\title{
Superconductivity and antiferromagnetism in quasi-one-dimensional organic conductors
}

\author{
(Review Article) \\ N. Dupuis ${ }^{1,2}$, C. Bourbonnais ${ }^{3}$, and J.C. Nickel ${ }^{2,3}$ \\ ${ }^{1}$ Department of Mathematics, Imperial College, 180 Queen's Gate, London SW7 $2 A Z$ \\ United Kingdom \\ E-mail: n.dupuis@imperial.ac.uk \\ ${ }^{2}$ Laboratoire de Physique des Solides, CNRS UMR 8502, Université Paris-Sud, 91405 Orsay, France \\ ${ }^{3}$ Regroupement Québecois sur les Matériaux de Pointe, Université de Sherbrooke \\ Sherbrooke, Québec, Canada J1K-2R1
}

Received September 15, 2005

\begin{abstract}
We review the current understanding of superconductivity in the quasi-one-dimensional organic conductors of the Bechgaard and Fabre salt families. We discuss the interplay between superconductivity, antiferromagnetism, and charge-density-wave fluctuations. The connection to recent experimental observations supporting unconventional pairing and the possibility of a triplet-spin order parameter for the superconducting phase is also presented.
\end{abstract}

PACS: 74.70.Kn, 74.20.Mn, 75.30.Fv

Keywords: superconductivity, antiferromagnetism, unconventional pairing.

\section{Introduction}

Superconductivity in organic conductors was first discovered in the ion radical salt (TMTSF) ${ }_{2} \mathrm{PF}_{6}$ [1]. Later on, it was found in most Bechgaard $\left[(\mathrm{TMTSF})_{2} \mathrm{X}\right]$ and Fabre $\left[(\mathrm{TMTTF})_{2} \mathrm{X}\right]$ salts. These salts are based on the organic molecules tetramethyltetraselenafulvalene (TMTSF) and tetramethyltetrathiafulvalene (TMTTF). The monovalent anion $\mathrm{X}$ can be either a centrosymmetric $\left(\mathrm{PF}_{6}, \mathrm{AsF}_{6}\right.$, etc.) or a non-centrosymmetric $\left(\mathrm{ClO}_{4}, \mathrm{ReO}_{4}, \mathrm{NO}_{3}\right.$, $\mathrm{FSO}_{3}, \mathrm{SCN}$, etc.) inorganic molecule. (See Refs. 2,3 for previous reviews on these compounds.) Although they are definitely not «high- $T_{c} »$ superconductors the transition temperature is of the order of $1 \mathrm{~K}-$, these quasi-one-dimensional (quasi-1D) conductors share several properties of high- $T_{c}$ superconductors and other strongly-correlated electron systems such as layered organic superconductors [4,5] or heavy-fermion materials [6]. The metallic phase of all these conductors exhibits unusual properties which cannot be explained within the framework of Landau's Fermi liquid theory and remain to a large extent to be understood. The superconducting phase is unconventional (not $s$-wave). Magnetism is ubiquitous in these correlated systems and might provide the key to the understanding of their behavior.

The quest for superconductivity in organic conductors was originally motivated by Little's proposal that highly polarizable molecules could lead - via an excitonic pairing mechanism - to tremendously large transition temperatures. Early efforts towards the chemical synthesis of such compounds were not successful, as far as superconductivity is concerned, but led to the realization of a $1 \mathrm{D}$ charge transfer salt (TTF-TCNQ) undergoing a Peierls instability at low temperatures [7]. Attempts to suppress the Peierls state and stabilize a conducting (and possibly superconducting) state by increasing the 3D character of this $1 \mathrm{D}$ conductor proved to be unsuccessful.

Organic superconductivity was eventually discovered in the the Bechgaard salt (TMTSF) ${ }_{2} \mathrm{PF}_{6}$ under $9 \mathrm{kbar}$ of pressure [1]. It was subsequently found in 
other members of the (TMTSF) ${ }_{2} \mathrm{X}$ series. Most of the Bechgaard salts are insulating at ambient pressure and low temperatures [8], and it came as a surprise that the insulating state of these materials is a spin-density-wave (SDW) rather than an ordinary Peierls state [2]. The important part played by magnetism in these compounds was further revealed when it was found that their phase diagram only shows a part of a larger sequence of ordered states, which includes the Néel and the spin-Peierls phases of their sulfur analogs, the Fabre salts (TMTTF) ${ }_{2} \mathrm{X}$ series [9].

The charge transfer from the organic molecules to the anions leads to a commensurate band filling $3 / 4$ coming from the 2:1 stoichiometry. The metallic character of these compounds at high enough temperature is due to the delocalization of carriers via the overlap of $\pi$-orbitals between neighboring molecules along the stacking direction ( $a$ axis) (Fig. 1) [2]. The electronic dispersion relation obtained from quantum chemistry calculations (extended Hückel method) is well approximated by the following tight-binding form [10-13]

$$
\begin{gathered}
\epsilon(\mathbf{k})=-2 t_{a} \cos \left(k_{a} a / 2\right)-2 t_{\perp b} \cos \left(k_{b} b\right)-2 t_{\perp c} \cos \left(k_{c} c\right) \simeq \\
\simeq v_{F}\left(\left|k_{a}\right|-k_{F}\right)-2 t_{\perp b} \cos \left(k_{b} b\right)-2 t_{\perp b}^{\prime} \cos \left(k_{b} b\right)- \\
-2 t_{\perp c} \cos \left(k_{c} c\right)+\mu,
\end{gathered}
$$
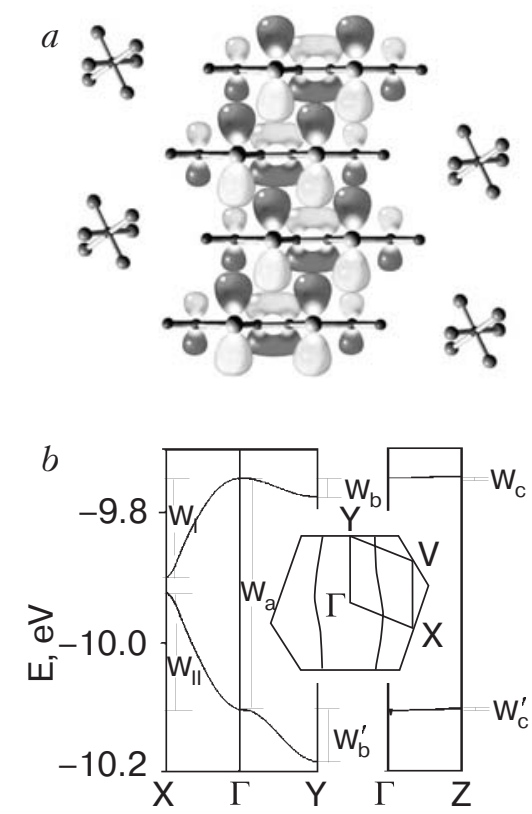

Fig. 1. A side view of the Bechgaard/Fabre salt crystal structure with the electron orbitals of the organic stacks (courtesy of J.Ch. Ricquier) ( $a$ ). Electronic dispersion relation and projected 2D Fermi surface of (TMTTF $)_{2} \mathrm{Br}$ (reprinted with permission from Ref. 13. Copyright 1994 by EDP Sciences) (b). where it is assumed that the underlying lattice is orthorhombic. This expression is a simplification of the dispersion relation - the actual crystal lattice symmetry is triclinic - but it retains the essential features. The conduction band along the chain direction has an overall width $4 t_{a}$ ranging between 0.4 and $1.2 \mathrm{eV}$, depending on the organic molecule (TMTSF or TMTTF) and the anion. As the electronic overlaps in the transverse $b$ and $c$ directions are much weaker than along the organic stacks, the dispersion law is strongly anisotropic, $t_{\perp b} / t_{a} \simeq 0.1$ and $t_{\perp c} / t_{\perp b} \simeq 0.03$, and the Fermi surface consists of two open warped sheets (Fig. 1). In the second line of Eq. (1), the electronic dispersion is linearized around the two 1D Fermi points $\pm k_{F}$, with $v_{F}$ the Fermi velocity along the chains ( $\mu$ is the chemical potential). The next-nearest-chain hopping $t^{\prime}{ }_{\perp b} \propto 2 t_{\perp b}^{2} / t_{a}+\ldots$ is introduced in order to keep the shape of the Fermi surface unchanged despite the linearization. The anions located in centrosymmetric cavities lie slightly above or below the molecular planes. This structure leads to a dimerization of the organic stacks and a (weak) gap $\Delta_{D}$, thus making the hole-like band effectively half-filled at sufficiently low energy or temperature [14,15]. (See Refs. 2,7,9 for a detailed discussion of the structural properties of quasi-1D organic conductors.) In the presence of interactions, commensurate band-filling introduces Umklapp scattering, which affects the nature of the possible phases in these materials.

What is remarkable about these electronic systems is the variety of ground states that can be achieved either by chemical means, namely substituting selenium by sulfur in the organic molecule or changing the nature of the anion (its size or symmetry), or applying pressure (Fig. 2). At low pressure, members of the sulfur series are Mott insulators (MI) from which either a lattice distorted spin-Peierls (SP) state - often preceded by a charge ordered (CO) state - or a commensurate-localized antiferromagnetic state (AF) can develop. On the other hand, itinerant antiferromagnetism (spin-density wave (SDW)) or superconductivity is found in the selenide series. Under pressure, the properties of the Fabre salts evolve towards those of the Bechgaard salts. The compound (TMTTF) ${ }_{2} \mathrm{PF}_{6}$ spans the entire phase diagram as pressure increases up to $50 \mathrm{kbar}$ or so (Fig. 3) [16-18], thus showing the universality of the phase diagram in Fig. 2 [19].

A large number of both theoretical and experimental works have been devoted to the understanding of the normal phase and the mechanisms leading to long-range order at low temperature. The presence of antiferromagnetism over a large pressure range does 


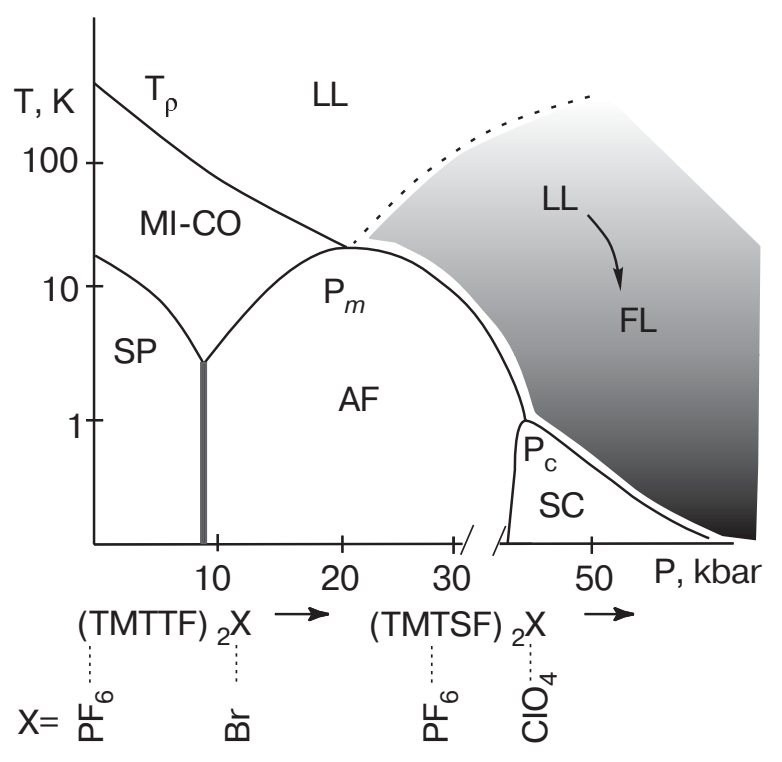

Fig. 2. The generic phase diagram of the Bechgaard/Fabre salts as a function of pressure or anion $\mathrm{X}$ substitution; Luttinger liquid (LL), Mott insulator (MI), charge order (CO), spin-peierls (SP), antiferromagnetism (AF), superconductivity (SC), Fermi liquid (FL).

indicate that repulsive interactions among carriers are important. The low-dimensionality of the system is also expected to play a crucial role. On the one hand, in the presence of repulsive interactions a strongly anisotropic Fermi surface with good nesting properties is predominantly unstable against the formation of an SDW state which is reinforced at low temperature by commensurate band filling. On the other hand, when the temperature exceeds the transverse dispersion $\sim t_{\perp b}, 3 \mathrm{D}$ (or 2D) coherent electronic motion is suppressed and the conductor behaves as if it were $1 \mathrm{D}$; the Fermi liquid picture breaks down and the system becomes a Luttinger liquid [20,21]. The relevance of 1D physics for the low-temperature properties $\left(T \lesssim t_{\perp b}\right)$, as well as a detailed description of the crossover from the Luttinger liquid to the Fermi liquid, is one of the most important issues in the debate surrounding the theoretical description of the normal state of these materials. As far as low-temperature phases are concerned, a chief objective is to reach a good description of the superconducting phase - the symmetry of the order parameter is still under debate - and the mechanisms leading to superconductivity. Owing to the close proximity of superconductivity and magnetism in the phase diagram of Fig. 2, it is essential to first discuss the origin of antiferromagnetism in both series of compounds.

\section{Néel antiferromagnetism and spin-density wave}

\subsection{Fabre salts at ambient pressure: Mott-insulator regime}

The Fabre salts (TMTTF) ${ }_{2} \mathrm{X}$ at ambient pressure are located on the left of the phase diagram in Fig. 2. Both the nature of correlations and the mechanism of long-range order at low temperature are now rather well understood. Below the temperature $T_{\rho} \sim 100 \mathrm{~K}$ (see Fig. 2), the resistivity develops a thermally activated behavior [22] and the system enters a Mott-insulator regime. The corresponding charge gap $\Delta_{\rho} \sim \pi T_{\rho}$ can be deduced from $T_{\rho}$ and turns out to be larger than the (bare) transverse bandwidth $t_{\perp b}$, which in turn suppresses any possibility of transverse single particle band motion and makes the system essentially one-dimensional. The charge gap $2 \Delta_{\rho}$ is also directly observed in the optical conductivity [23]. The members of the (TMTTF) $)_{2} \mathrm{X}$ series thus behave as typical 1D Mott insulators below $T_{\rho}$ with the carriers confined along the organic stacks - as a result of the Umklapp scattering due to the commensurability of the electronic density with the underlying lattice $[14,15]$. This interpretation agrees with the absence of anomaly in the spin susceptibility at $T_{\rho}$ [24], in accordance with the spin-charge separation characteristic of 1D systems [21]. It is further confirmed by measurements of the spin-lattice relaxation rate $1 / T_{1}$. The Luttinger liquid theory predicts $[25,26]$

$$
\frac{1}{T_{1}}=C_{0} T \chi_{s}^{2}(T)+C_{1} T^{K_{\mathrm{p}}},
$$

where $C_{0}$ and $C_{1}$ are temperature independent constants. $\chi_{s}(T)$ is the uniform susceptibility and $K_{\rho}$ the Luttinger liquid charge stiffness parameter. The two contributions in (2) correspond to paramagnons or spinons $(q \simeq 0)$ and AF spin fluctuations $\left(q \simeq 2 k_{F}\right)$. Both the temperature dependence of $\chi_{s}(T)$ and the presence of AF fluctuations lead to an enhancement of $1 / T_{1}$ with respect to the Korringa law $\left(T_{1} T\right)^{-1}=$ const which holds in higher-dimensional metals. In a 1D Mott insulator $K_{\rho}=0$, which leads to $T_{1}^{-1}=C_{0} T \chi_{s}^{2}(T)+C_{1}$ in good agreement with experimental measurements of $T_{1}$ and $\chi_{s}$ [24].

The low-energy excitations in the Mott-insulator regime are $1 \mathrm{D}$ spin fluctuations. By lowering the temperature, these fluctuations can propagate in the transverse direction and eventually drive an AF transition. This transition is not connected to Fermi surface effects. The condition $\Delta_{\rho}>t_{\perp b}$ precludes a single-particle coherent motion in the transverse direction, and the concept of Fermi surface remains ill defined in the Fabre salts at ambient pressure. AF 
long-range order comes from interchain transfer of bound electron-hole pairs leading to a kinetic exchange interaction $J_{\perp}$ between spin densities on neighboring chains - much in analogy with the exchange interaction between localized spins in the Heisenberg limit. An effective Hamiltonian can be derived from a renormalization group (RG) calculation, [27,28]

$$
H_{\perp}=J_{\perp} \int d x \sum_{\langle i, j\rangle} \mathbf{S}_{i}(x) \cdot \mathbf{S}_{j}(x), \quad J_{\perp} \simeq \frac{\xi_{\rho}}{a} \frac{t_{\perp b}^{* 2}}{\Delta_{\rho}}
$$

where $t_{\perp b}^{*}$ is the effective interchain hopping at the energy scale $\Delta_{\rho}$ and $a$ the lattice spacing along the chain. The sum in Eq. (3) is over nearest-neighbor chains. The naive value $t_{\perp b}^{* 2} / \Delta_{\rho}$ of the exchange interaction $J_{\perp}$ is enhanced by the factor $\xi_{\rho} / a$ where $\xi_{\rho}=v_{F} / \Delta_{\rho}$ is the intrachain coherence length induced by the Mott gap along which virtual interchain hoppings can take place. Within a mean-field treatment of $H_{\perp}$, the condition for the onset of long-range order is given by $J_{\perp} \chi_{1 \mathrm{D}}\left(2 k_{F}, T\right)=1$ where $\chi_{1 \mathrm{D}}\left(2 k_{F}, T\right) \sim\left(T / \Delta_{\rho}\right)^{-1}$ is the exact power law form of the 1D AF spin susceptibility. This yields a Néel temperature

$$
T_{N} \sim \frac{t_{\perp b}^{* 2}}{\Delta_{\rho}} .
$$

Since $T_{\rho}$ and $\Delta_{\rho}$ decrease under pressure (Fig. 2), Eq. (4) predicts an increase of $T_{N}$ with pressure - assuming a weak pressure dependence of $t_{b}^{*}-$ as observed experimentally (see Fig. 2). The relation $T_{N} T_{\rho} \sim t_{\perp b}^{* 2} \sim$ const has been observed in (TMTTF) ${ }_{2} \mathrm{Br}[29]$.

\subsection{Bechgaard salts: itinerant magnetism}

With increasing pressure, $T_{\rho}$ drops and finally merges with the $\mathrm{AF}$ transition line at $P_{m}$, beyond which there is no sign of a Mott gap in the normal phase. The Fabre salts then tend to behave similarly to the Bechgaard salts (Fig. 2). The change of behavior at $P_{m}$ is usually attributed to a deconfinement of carriers, i.e., a crossover from a Mott insulator to a - metallic - Luttinger liquid. At lower temperature, single-particle transverse hopping is expected to become relevant and induces a dimensional crossover at a temperature $T_{x}$ from the Luttinger liquid to a $2 \mathrm{D}$ or $3 \mathrm{D}$ metallic state. With increasing pressure, the AF transition becomes predominantly driven by the instability of the whole warped Fermi surface due to the nesting mechanism. Although there is a general agreement on this scenario, there is considerable debate on how the dimensional crossover takes place and the nature of the low-temperature metallic state.
On the theoretical side, simple RG arguments indicate that the crossover from the Luttinger liquid to the $2 \mathrm{D}$ regime takes place at the temperature [30]

$$
T_{x} \sim \frac{t_{\perp b}}{\pi}\left(\frac{t_{\perp b}}{t_{a}}\right)^{\frac{1-K_{\mathrm{p}}}{K_{\mathrm{p}}}}
$$

where $K_{\rho}$ is the Luttinger liquid parameter. For non-interacting electrons $\left(K_{\rho}=1\right)$, Eq. (5) would give $T_{x} \sim t_{\perp b}$ : the 2D Fermi surface is irrelevant when temperature is larger than the dispersion in the $b$ direction. For interacting electrons $\left(K_{\rho} \neq 1\right)$, the interchain hopping amplitude $t_{\perp b}$ is reduced to an effective value $t_{\perp b}$ and the dimensional crossover occurs at a lower temperature $T_{x} \sim t_{\perp b}^{*}<t_{\perp b}$. A detailed theoretical picture of the dimensional crossover is still lacking. In particular, whether it is a sharp crossover or rather extends over a wide temperature range - as shown by the shaded area in Fig. 2 - is still an open issue.

\subsubsection{The strong-correlation picture}

Some experiments seem to indicate that correlations still play an important role even in the low-temperature phase of the Bechgaard salts. For instance, a significant enhancement of $1 / T_{1} T$ with respect to the Korringa law - although weaker than in the Fabre salts at ambient pressure - is still present [24]. This behavior has been explained in terms of $1 \mathrm{D}$ spin fluctuations persisting down to the dimensional crossover temperature $T_{x} \sim 10 \mathrm{~K}$, below which the Korringa law is recovered $[24,26]$.

The restoration of a plasma edge in the transverse $b^{\prime}$ direction at low temperature in (TMTSF) ${ }_{2} \mathrm{PF}_{6}-\mathrm{ab}-$ sent in the Fabre salts - suggests the gradual emergence of a coherent motion in the $(a b)$ planes below $T_{x} \sim 100 \mathrm{~K}[31,32]$. ( $\mathbf{b}^{\prime}$ is normal to $\mathbf{a}$ and $\mathbf{c}$ in the (ab) plane. It differs from $\mathbf{b}$ due to the triclinic structure.) However, the frequency dependence of the optical conductivity is inconsistent with a Drude-like metallic state $[33,34,23]$. The low-energy peak carries only $1 \%$ of the total spectral weight and is too narrow to be interpreted as a Drude peak with a frequency-independent scattering time. It has been proposed that this peak is due to a collective mode that bears some similarities with the sliding of a charge-density wave - an interpretation supported by the new phonon features that emerge at low temperature [33]. Furthermore, $99 \%$ of the total spectral weight is found in a finite energy peak around $200 \mathrm{~cm}^{-1}$. It has been suggested that this peak is a remnant of a (1/4)-filled Mott gap $\Delta_{\rho}$, observed in the less metallic Fabre salts at ambient pressure [35,23]. In this picture, $(\mathrm{TMTSF})_{2} \mathrm{PF}_{6}$ is close to the border between a Mott insulator and a Luttinger liquid, and the low-temperature metallic 
behavior is made possible by the interchain coupling [23,36,37]. A different interpretation has been proposed for the far infrared spectrum in optical conductivity and is based on the weak half-filling character of the band for interactions in the Hubbard limit [38].

The longitudinal resistivity in (TMTSF) ${ }_{2} \mathrm{PF}_{6}$ is found to be metallic, with a $T^{2}$ law between the SDW transition and $150 \mathrm{~K}$, crossing over to a sublinear temperature dependence above $150 \mathrm{~K}$ with an exponent in the range $0.5-1[39,40]$. While this observation would be consistent with a dimensional crossover to a low-temperature Fermi liquid regime taking place at $T_{x} \sim 150 \mathrm{~K}$, the transverse resistance $\rho_{b}$ along the $b$ axis apparently fails to show the expected $T^{2}$ behavior. Given the difficulties of a direct dc measurement, owing to non-uniform current distributions between contacts, conflicting results have been published in the literature [39,41]. Nevertheless, below $T \sim 80 \mathrm{~K}$ $\rho_{b}$ can be deduced from $\rho_{a} \sim T^{2}$ and $\rho_{c} \sim T^{1.5}$ using a tunneling argument, which yields $\rho_{c}=\left(\rho_{a} \rho_{b}\right)^{1 / 2}$ and therefore $\rho_{b} \sim T$. Moreover, contactless - microwave - transverse conductivity measurements in the (TMTSF) ${ }_{2} \mathrm{PF}_{6}$ salt fail to reveal the emergence of a Fermi liquid $T^{2}$ temperature dependence of the resistivity in the $b$ direction in this temperature range [42]. As far as $\rho_{c}$ is concerned, a maximum around $T_{\max } \sim 80 \mathrm{~K}$ has been observed, with a metallic though incoherent - behavior $\rho_{c} \sim T^{1.5}$ at lower temperature [43]. $T_{\max }$ is highly sensitive to pressure, whereas the interchain hopping $t_{\perp b}$ is not. Therefore, $T_{\max }$ cannot be directly identified with $t_{\perp b}$, but could be related to a - weakly - renormalized value $t_{\perp b}^{*} \sim T_{x}$ in agreement with predictions of the Luttinger liquid theory [see Eq. (5)]. The transport measurements seem to be indicative of a gradual crossover between a Luttinger liquid and a Fermi liquid occurring in the temperature range $40-80 \mathrm{~K}$. The onset of 3D coherence and Fermi liquid behavior would then be related to the interplane coupling $t_{\perp c}$ between $(a, b)$ planes [43].

The absence of Fermi liquid behavior down to very low temperatures in the Bechgaard salts seems to be further supported by photoemission experiments. ARPES fails to detect quasi-particle features or the trace of a Fermi surface at 150 K [44]. Similar conclusions were deduced from integrated photoemission at $50 \mathrm{~K}$ [45]. However, photoemission results - e.g. the absence of dispersing structure and a power-law frequency dependence which is spread over a large energy scale of the order of $1 \mathrm{eV}$ - do not conform with the predictions of the Luttinger theory and might be strongly influenced by surface effects.

The existence of strong correlations suggests that the kinetic interchain exchange $J_{\perp}$, which drives the
AF transition in the sulfur series, still plays an important role in the Bechgaard salts. In this picture, the decrease of $T_{N}$ with increasing pressure is due both to the decrease of $J_{\perp}$ and the deterioration of the Fermi surface nesting. This scenario is supported by RG calculations [28].

All the experiments mentioned so far favor different - and sometimes incompatible - scenarios for the dimensional crossover. However, the high-temperature phase of the Bechgaard salts is always analyzed on the basis of the Luttinger liquid theory. A consistent interpretation of the experimental results therefore requires to find a common $K_{\rho}$ parameter and to determine the value of the remnant of the Mott gap $\Delta_{\rho}$. NMR [24], dc transport [43,46], and optical measurements $[23,36]$ have been interpreted in terms of the Luttinger theory with $K_{\rho} \simeq 0.23$ and quarter-filled Umklapp scattering $[7,37]$. This interpretation, as well as the mere existence of strong correlations, is not without raising a number of unanswered questions (see the next section). For instance, $K_{\rho} \simeq 0.23$ would lead according to (5) to $T_{x} \sim 10^{-3} t_{\perp b}$, a value much below the experimental observations.

\subsubsection{The weak-correlation picture}

On the other hand, there are experiments pointing to the absence of strong correlations in the Bechgaard salts. One of the most convincing arguments comes from the so-called Danner-Chaikin oscillations [47]. Resistance measurements of (TMTSF) ${ }_{2} \mathrm{ClO}_{4}$ in the $c$ direction show pronounced resonances when an applied magnetic field is rotated in the (ac) plane at low temperature. The complete angular dependence of the magneto-resistance can be reproduced within a semiclassical approach. The position of the resonance peaks is given by the zeros of the Bessel function $J_{0}(\gamma)$ evaluated at $\gamma=2 t_{\perp b} c B_{x} / v_{F} B_{z}$ (c is the interchain spacing in the $c$ direction). This enables a direct measure of the interchain hopping amplitude in the $b$ direction, yielding $t_{\perp b} \simeq 280 \mathrm{~K}$ above the anion ordering transition taking place at $24 \mathrm{~K}$, in very good agreement with values derived from band calculations [10-12]. These results can hardly be reconciled with the existence of strong correlations. Sizeable 1D fluctuations should lead to a strong $\left(k_{\|}, w\right)$ dependence of the self-energy, and in turn to a significant renormalization of $k_{\perp}$-dependent quantities like the interchain hopping amplitudes [28]. This lends support to the idea that the low-temperature phase of the Bechgaard salts can be described as a weakly interacting Fermi liquid subject to spin fluctuations induced by the nesting of the Fermi surface $[48,49]$. 
The weak-coupling approach has been particularly successful in the framework of the Quantized Nesting Model [50-52]. The latter explains the cascade of SDW phases induced by a magnetic field in $(\mathrm{TMTSF})_{2} \mathrm{PF}_{6}$ and $(\mathrm{TMTSF})_{2} \mathrm{ClO}_{4}$, and provides a natural explanation for the quantization of the Hall effect $-\sigma_{x y}=2 N e^{2} / h$ ( $N$ integer) per $(a b)$ plane observed in these phases. Furthermore, it reproduces the experimental phase diagram only for interchain hopping amplitudes $t_{\perp b}, t_{\perp c}$ close to their unrenormalized values.

Despite the apparent success of the weak-coupling approach, it has nevertheless become clear that the SDW phase of the Bechgaard salts is not conventional. Recent experiments have shown that the $2 k_{F}$ SDW coexists with a $2 k_{F}$ and a - weaker $-4 k_{F}$ charge-density wave (CDW) in (TMTSF) ${ }_{2} \mathrm{PF}_{6}$ $[53,54]$. Since there is no $2 k_{F}$ phonon softening associated to this transition, the emergence of this CDW state differs from what is usually seen for an ordinary Peierls state. This unusual ground-state can be explained on the basis of a quarter-filled 1D model with dimerization and onsite, nearest-neighbor and next-nearest-neighbor Coulomb interactions [55-59], but this explanation remains to be confirmed.

\subsubsection{The normal phase above the superconducting phase}

It is remarkable that the superconducting phase lies next to the SDW phase - which is actually a mixture SDW-CDW - and reaches its maximum transition temperature $T_{c} \sim 1 \mathrm{~K}$ at the pressure $P_{c}$ where $T_{S D W}$ and $T_{c}$ join (see Figs. 2 and 3 ). In the normal phase above the SDW phase, the resistivity along the $a$ axis decreases with temperature, reaches a minimum at $T_{\min }$, and then shows an upturn and a strong enhancement related to the proximity of the SDW phase transition that occurs at $T_{S D W}<T_{\min }$. The region of the normal phase where strong AF fluctuations are present $\left(T_{S D W}<T<T_{\min }\right)$ extends over the pressure range where the ground state is superconducting (Fig. 3). Its width in temperature decreases with increasing pressure, so that the superconducting transition temperature appears to be closely linked to $T_{\min }$. These observations strongly suggest an intimate relationship between spin fluctuations and superconductivity in the Bechgaard/Fabre salts $[16,17]$. The importance of spin fluctuations above the superconducting phase is further confirmed by the persistence of the enhancement of the spin-lattice relaxation rate $1 / T_{1}$ for $P>P_{C}$ [24]. Besides the presence of spin fluctuations at low temperature, charge fluctuations have also been observed in the normal phase via optical conductivity measurements [33].

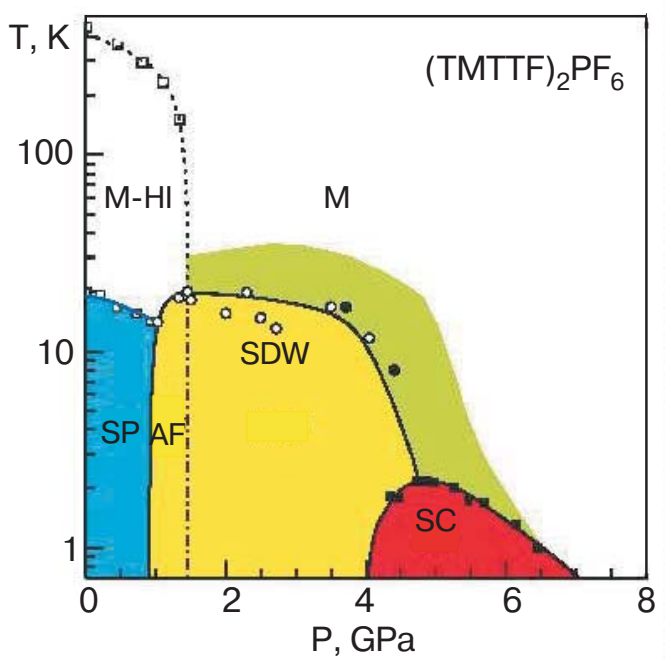

Fig. 3. $(P, T)$ phase diagram of (TMTTF $)_{2} \mathrm{PF}_{6}$. The shaded area above the SDW and SC phase indicates the region of the normal phase where spin fluctuations are significant. (Reprinted with permission from Ref. 17. Copyright 2001 by EDP Sciences.)

\section{Superconductivity}

Some of the early experiments in the Bechgaard salts were not in contradiction with a conventional BCS superconducting state. For instance, the specific heat in (TMTSF) ${ }_{2} \mathrm{ClO}_{4}$ obeys the standard temperature dependence $C / T=\gamma+\beta T^{2}$ above the superconducting transition, and the jump at the transition $\Delta C / \gamma T_{c} \simeq 1.67$ is close to the BCS value 1.43 . The ratio $2 \Delta(T=0) / T_{C} \simeq 3.33$, obtained from the gap deduced from the thermodynamical critical field, is also in reasonable agreement with the prediction of the BCS theory $\left(2 \Delta / T_{c} \simeq 3.52\right)[60,61]$. Early measurements of $H_{c 2}(T)$, performed in the vicinity of the zero-field transition temperature, were also interpreted on the basis of the BCS theory [62-65].

Nevertheless, soon after the discovery of organic superconductivity, the high-sensitivity of the superconducting state to irradiation [66,67] led Abrikosov [68] to suggest the possibility of an unconventional - triplet - pairing, although the non-magnetic nature of the induced defects is questionable [7]. The sensitivity to non-magnetic impurities, and thus the existence of unconventional pairing, was later on clearly established by the suppression of the superconducting transition upon alloying (TMTSF) ${ }_{2} \mathrm{ClO}_{4}$ with a very small concentration of $\mathrm{ReO}_{4}$ anions [69,70]. A recent study [71] of the alloy (TMTSF $)_{2}\left(\mathrm{ClO}_{4}\right)_{x}\left(\mathrm{ReO}_{4}\right)_{1-x}$ - with different cooling rates and different values of $x$ - has confirmed this in remarkable way by showing 
that the transition temperature $T_{c}$ is related to the scattering rate $1 / \tau$ by

$$
\ln \left(\frac{T_{c 0}}{T_{c}}\right)=\Psi\left(\frac{1}{2}+\frac{1}{4 \pi \tau T_{c}}\right)-\Psi\left(\frac{1}{2}\right)
$$

( $T_{c 0}$ is the transition temperature of the pure system and $\Psi$ the digamma function), as expected for an unconventional superconductor in the presence of non-magnetic impurities [72].

Another indication of a possible unconventional pairing came from the observation of Gor'kov and Jérome [73] that the upper critical field $H_{c 2}(T)$, extrapolated down to $T=0$, would exceed the Pauli limited field [74,75] $H_{P}=1.84 T_{c 0} / \mu_{B} \sim 2 \mathrm{~T}$ by a factor of 2. (The value of $H_{P}$ quoted here corresponds to $s$-wave pairing.) As spin-orbit interaction is weak in these systems and cannot provide an explanation for such a large $H_{c 2}$, it is tempting to again invoke triplet pairing. This issue has been revived by recent measurements of the upper critical field in (TMTSF) ${ }_{2} \mathrm{PF}_{6}$ with substantially improved accuracy in angular alignment and lower temperatures. Lee et al. [76,77] observed a pronounced upward curvature of $H_{c 2}(T)$ without saturation - down to $T \sim T_{c} / 60-$ for a field parallel to the $a$ or $b^{\prime}$ axis, with $H_{c 2}^{b^{\prime}}(T)$ and $H_{c 2}^{a}(T)$ exceeding the Pauli limited field $H_{P}$ by a factor of 4. Moreover, $H_{c 2}^{b^{\prime}}(T)$ becomes larger than $H_{c 2}^{a}(T)$ at low temperatures. Similar results were obtained from simultaneous resistivity and torque magnetization experiments in (TMTSF) ${ }_{2} \mathrm{ClO}_{4}$ (Fig. 4)

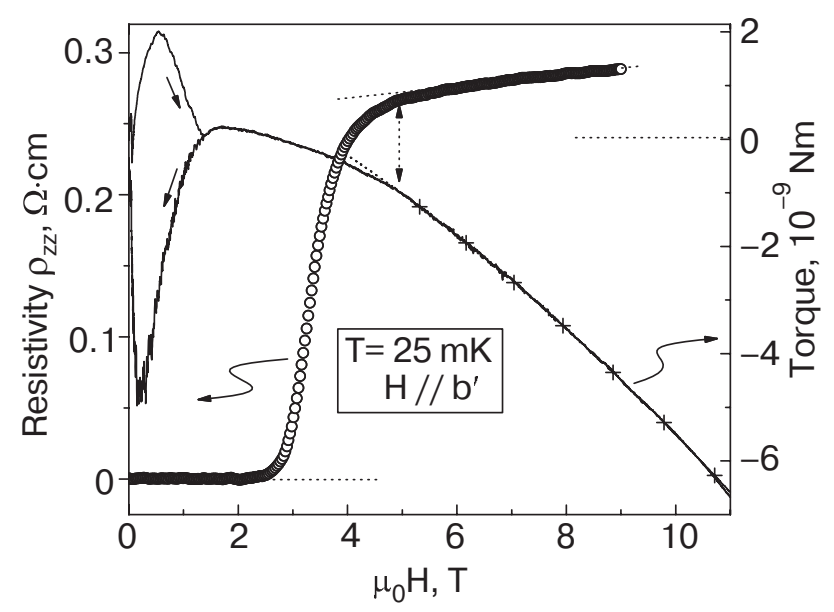

Fig. 4. Resistivity (left scale) and torque magnetization (right) in (TMTSF) ${ }_{2} \mathrm{ClO}_{4}$ at $25 \mathrm{mK}$ for $H \| b^{\prime}$. The dotted line and + symbols on the torque curve represent a temperature-independent normal state contribution. The onsets of diamagnetism and decreasing resistivity, upon decreasing field, are indicated by the arrow near $H_{c 2} \sim 5 \mathrm{~T}$. Arrows in the low field vortex state indicate field sweep directions. (Reprinted with permission from Ref. 78. Copyright 2004 by the American Physical Society.)
[78]. The extrapolated value to zero temperature, $H_{c 2}(0) \sim 5 \mathrm{~T}$, is at least twice the Pauli limited field.

There are different mechanisms that can greatly increase the orbital critical field $H_{c 2}^{\text {orb }}(T)$ in organic conductors. Superconductivity in a weakly-coupled plane system can survive in a strong parallel magnetic field if the interplane (zero-field) coherence length $\xi_{\perp}(T)$ becomes smaller than the interplane spacing $d$ at low temperature. Vortex cores, with size $\xi_{\perp}(T) \lesssim d$, can then fit between planes without destroying the superconducting order in the planes, and lead to a Josephson vortex lattice. In the Bechgaard salts, even for a field parallel to the $b^{\prime}$ axis, the Josephson limit $\xi_{\perp}(T) \lesssim d$ is however unlikely to be reached, since the interchain hopping amplitude $t_{\perp c} \sim 5-10 \mathrm{~K}$ is larger than the transition temperature $T_{c} \sim 1.1 \mathrm{~K}$. Nevertheless the orbital critical field can be enhanced by a field-induced dimensional crossover [79-83]. A magnetic field parallel to the $b^{\prime}$ axis tends to localize the wavefunctions in the $(a c)$ planes, which in turn weakens the orbital destruction of the superconducting order. When $\omega_{c}=e H c \gtrsim t_{\perp c}$ (which corresponds to a field of a few Tesla in the Bechgaard salts), the wave functions are essentially confined in the $(a c)$ planes and the orbital effect of the field is completely suppressed. The coexistence between SDW and superconductivity, as observed in a narrow pressure domain of the order of 0.8 kbar below the critical pressure $P_{c}$ (Fig. 2), can also lead to a large increase of the orbital upper critical field [84-88].

Regardless of the origin of the large orbital critical field, another mechanism is required to exceed the Pauli limited field $H_{P}$ in the Bechgaard salts. For singlet spin pairing, the Pauli limit may be overcome by a non-uniform Larkin-Ovchinnikov-Fulde-Ferrell (LOFF) state, where Cooper pairs form with a nonzero total momentum $[89,90]$. This mechanism is particularly efficient in a $1 \mathrm{D}$ system $[79,81,83,91]$, due to the large phase space available for pairing at nonzero total momentum. For a linearized dispersion law, the mean-field upper critical field $H_{c}^{L O F F}$ diverges as $1 / T$ in a pure superconductor. Lebed [92] has argued that the quasi-1D anisotropy reduces $H_{c}^{L O F F}$ below the experimental observations. The only possible explanation for a large upper critical field would then be an equal-spin triplet pairing. A $p_{x}$-wave triplet state with a d vector perpendicular to the $b^{\prime}$ axis was proposed [93] as a possible explanation of the experimental observations reported in Refs. 76,77.

The triplet scenario in the Bechgaard salts is supported by recent NMR Knight shift experiments (Fig. 5) [94,95]. Early NMR experiments by Takigawa $e t$ al. already pointed to the unconventional nature of the superconducting state in (TMTSF) ${ }_{2} \mathrm{ClO}_{4}$ [96]. 


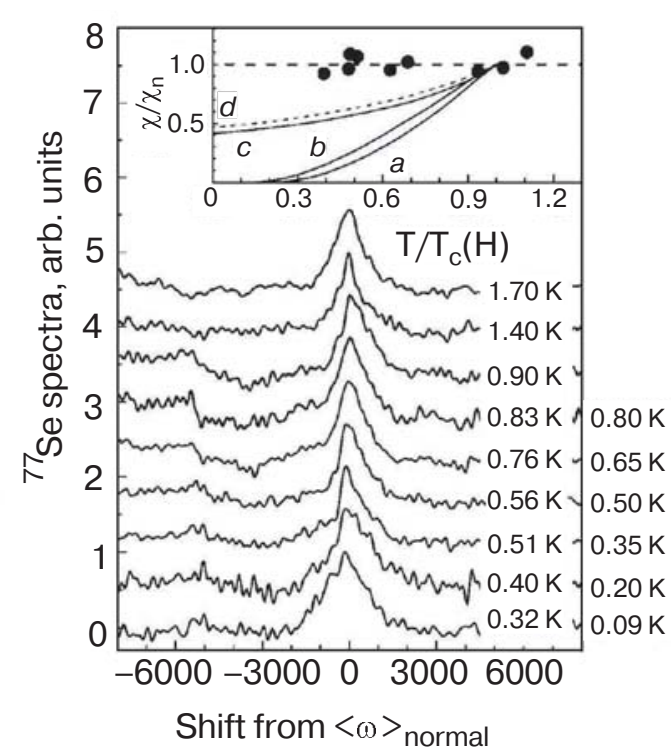

Fig. $5 .{ }^{77}$ Se NMR spectra $T_{c}(0.81 \mathrm{~K}$ at $1.43 \mathrm{~T})$. Each trace is normalized and offset for clarity. The temperatures shown in parentheses are the measured equilibrium temperatures before the pulse. In the inset, the spin susceptibility normalized by the normal state $\chi / \chi_{n}$ from measured first moments are compared with theoretical calculations [98] for $H / H_{c 2}(0) \sim 0(a)$ and $0.63(b)$. Curves $c$ and $d$ are obtained from the ratio of applied field $(1.43 \mathrm{~T})$ to the measured upper critical field $H_{c 2}(\mathrm{~T})$ at which the superconducting criteria «onset» and «50 transition» have been used, respectively, to determine $H_{c 2}(\mathrm{~T})$. (Reprinted with permission from Ref. 94. Copyright 2002 by the American Physical Society.)

The proton spin lattice relaxation rate $1 / T_{1}$ does not exhibit a Hebel-Slichter peak. It decreases rapidly just below $T_{C}$ in contrast to the typical BCS superconductor where it increases below $T_{c}$, reaching a maximum at $T \sim 0.9 T_{c}$. Furthermore, $1 / T_{1} \sim T^{3}$ for $T_{c} / 2 \lesssim T \leq T_{C}-$ as it is the case for most unconventional superconductors - suggesting zeros or lines of zeros in the excitation spectrum. Recent experiments by Lee et al. in (TMTSF) ${ }_{2} \mathrm{PF}_{6}$ show that the Knight shift, and therefore the spin susceptibility, remains unchanged at the superconducting transition [94,95]. This indicates triplet spin pairing, since a singlet pairing would inevitably lead to a strong reduction of the spin susceptibility $(\chi(T \rightarrow 0) \rightarrow 0)$. It should however be noticed that the interpretation of the Knight shift results - due to a possible lack of sample thermalization during the time of the experiment - has been questioned [7,97].

In principle, the symmetry of the order parameter can be determined from tunneling spectroscopy. Sign changes of the pairing potential around the Fermi surface lead to zero-energy bound states in the superconducting gap [98]. These states manifest themselves as a zero-bias peak in the tunneling conductance into the corresponding edge [99]. More generally, different pairing symmetries can be unambiguously distinguished by tunneling spectroscopy in a magnetic field [100-102]. In practice however, the realization of tunnel junctions with the TMTSF salts appears to be very difficult. A large zero-bias conductance peak - suggesting $p$-wave symmetry - across the junction between two organic superconductors was observed [103]. But the absence of temperature broadening could indicate that this peak is due to disorder rather than to a midgap state [104].

Information about the symmetry of the order parameter can also be obtained from thermal conductivity measurements. The latter indicate the absence of nodes in the excitation spectrum of the superconducting state in (TMTSF) ${ }_{2} \mathrm{ClO}_{4}[105]$, thus suggesting a $p_{x}$-wave symmetry. However, because of the doubling of the Fermi surface in the presence of anion ordering, a singlet $d$ - or triplet $f$-wave order phase would also be nodeless in (TMTSF) ${ }_{2} \mathrm{ClO}_{4}$ (see Fig. 6 for the different gap symmetries in a quasi-1D superconductor) $[9,106]$.

\section{Microscopic theories of the superconducting state}

The phase diagram of the 1D electron gas within the $g$-ology framework [108] is shown in Fig. 7. $g_{1}$ and $g_{2}$ denote the backward and forward scattering amplitudes, respectively, and $g_{3}$ the strength of the (half-filling) Umklapp processes. Given the importance of spin fluctuations in the phase diagram of the Bechgaard/Fabre salts, as well as the existence of AF
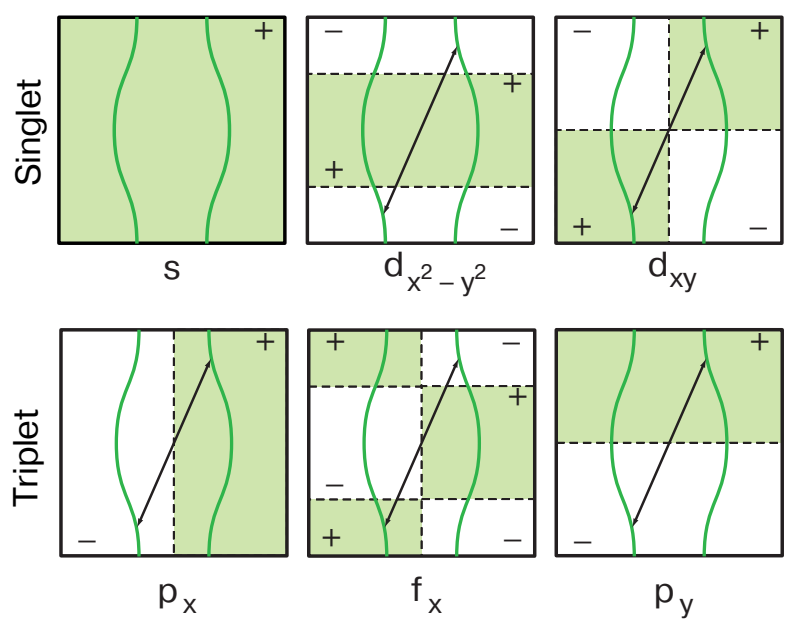

Fig. 6. Gap symmetries $\Delta_{r}\left(k_{\perp}\right)$ in a quasi-1D superconductor (after Ref. 107, courtesy of Y. Suzumura). $r=+/-$ denotes the right/left sheet of the Fermi surface. (Singlet pairing) $s$ : const, $d_{x^{2}-y^{2}}: \cos k_{\perp}, d_{x y}: r \sin k_{\perp}$. (Triplet pairing) $p_{x}: r, f: r \cos k_{\perp}, p_{y}: \sin k_{\perp}$. Next-nearest-neighbor and longer-range pairings are not considered. 
ground states, the Bechgaard/Fabre salts should pertain to the upper right corner of the $1 \mathrm{D}$ phase diagram $\left(g_{1}, g_{2}>0\right.$ and $\left.g_{1}-2 g_{2}<\left|g_{3}\right|\right)$ where the Umklapp processes are relevant and the dominant fluctuations antiferromagnetic. In the Fabre salts, the non-magnetic insulating phase observed below $T_{\rho} \sim 100 \mathrm{~K}$ indicates the importance of Umklapp scattering and suggests sizable values of $g_{3}$ for this series. Since the long-range Coulomb interaction favors $g_{1}<g_{2}$, the Fabre salts are expected to lie to the right of the phase diagram, i.e. far away from the boundary $g_{1}-2 g_{2}=\left|g_{3}\right|$. Since the triplet superconducting phase is lying next to the SDW phase (Fig. 7), it is tempting to invoke a change of the couplings $g_{i}$ under pressure to argue in favor of a $p_{x}$-wave triplet superconducting state $[68,109]$. Such a drastic change of the couplings, which would explain why (TMTTF) ${ }_{2} \mathrm{PF}_{6}$ becomes superconducting above $4.35 \mathrm{GPa}$ [16-18], is however somewhat unrealistic and has not received any theoretical backing so far. The Umklapp scattering being much weaker in the Bechgaard salts, one cannot exclude that these compounds lie closer to the boundary between the SDW and the triplet superconducting phase. A moderate change of the couplings under pressure would then be sufficient to explain the superconducting phase of (TMTSF) ${ }_{2} \mathrm{PF}_{6}$ observed above $6 \mathrm{kbar}$ or so. However, the destruction of the superconducting phase by a weak magnetic field and the observation of a cascade of SDW phases for slightly higher fields [50-52] would imply that the interaction is strongly magnetic-field dependent - again a very unlikely scenario.

In all probability, the very origin of the superconducting instability lies in the 3D behavior of these quasi-1D conductors. Thus the attractive interaction

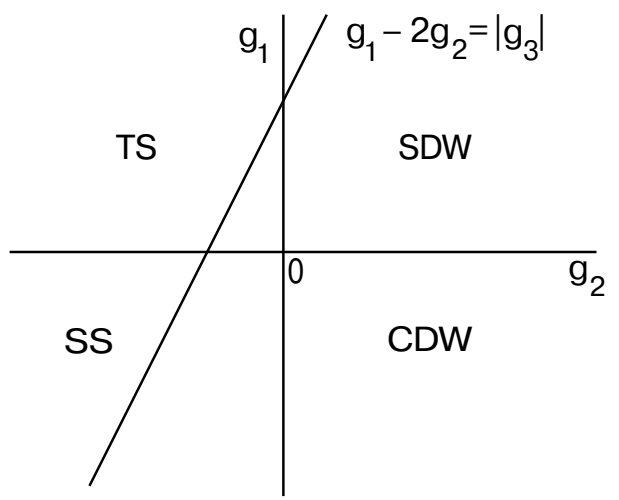

Fig. 7. Phase diagram (leading fluctuations) of the 1D electron gas in presence of Umklapp scattering. SS (TS): singlet (triplet) superconductivity. A gap develops in the charge sector (Mott insulating behavior) for $g_{1}-2 g_{2}<\left|g_{3}\right|$. is a consequence of a low-energy mechanism that becomes more effective below the dimensional crossover temperature $T_{x}$. Transverse hopping makes retarded electron-phonon interactions more effective, since it is easier for the electrons to avoid the Coulomb repulsion [109]. By comparing the sulfur and selenide series, it can however be argued that, in the pressure range where superconductivity is observed, the strength of the electron-phonon interaction is too weak to explain the origin of the attractive interaction. For narrow tight-binding bands in the organics, the attraction is strongest for backscattering processes in which $2 k_{F}$ phonons are exchanged [110,111]. According to the results of $x$-ray experiments performed on (TMTSF $)_{2} \mathrm{X}$, however, the electron-phonon vertex at this wave vector does not undergo any significant increase in the normal state (Fig. 8). The amplitude of the $2 k_{F}$ lattice susceptibility in (TMTSF) ${ }_{2} \mathrm{PF}_{6}$ - which is directly involved in the strength of the phonon exchange - is weak. It is instructive to compare with the sulfur analog compound (TMTTF) ${ }_{2} \mathrm{PF}_{6}$, for which the electron-phonon vertex at $2 k_{F}$ becomes singular, signaling a lattice instability towards a spin-Peierls distortion (Fig. 8). This instability produces a spin gap that is clearly visible in the temperature dependence of the magnetic susceptibility and nuclear relaxation rate $[112,113]$. These effects are not seen in (TMTSF) ${ }_{2} \mathrm{PF}_{6}$ close to $P_{c}$. The persistent enhancement of these quantities indicates that interactions are dominantly repulsive (Sec. 2.2.3.), making the traditional phonon-mediated source of pairing inoperant.

Emery [114] pointed out that near an SDW instability, short-range AF spin fluctuations can give rise to anisotropic pairing and thus provide a possible

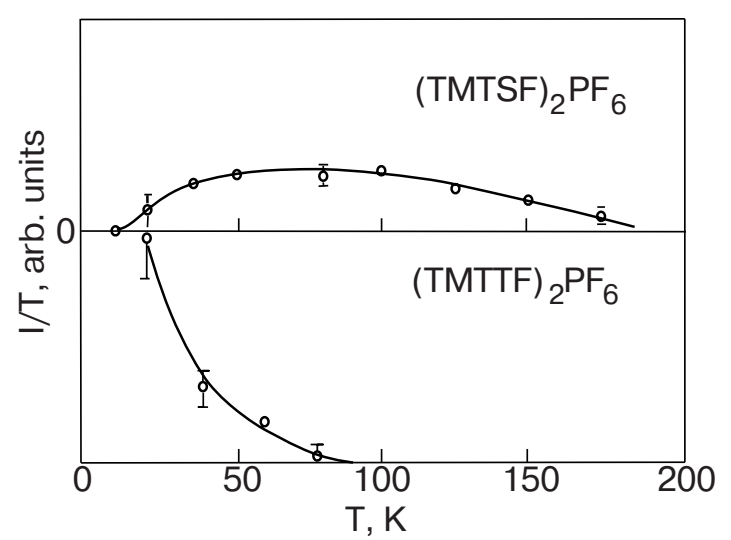

Fig. 8. Temperature dependence of the $2 k_{F}$ lattice susceptibility $(I / T)$ as a function of temperature in the normal phase of (TMTSF) ${ }_{2} \mathrm{PF}_{6}$ (top) and (TMTTF) ${ }_{2} \mathrm{PF}_{6}$ (bottom). (Reprinted with permission from Ref. 53. Copyright 1996 by EDP Sciences.) 
explanation of the origin of the superconducting phase in the Bechgaard salts. Such fluctuations give rise to an oscillating potential that couples to the electrons. Carriers can avoid the local Coulomb repulsion and take advantage of the attractive part of this potential by moving on different chains. This mechanism, which can lead to superconductivity at low temperatures, is the spin-analog of the so-called Kohn-Luttinger mechanism which assumes the pairing to originate in the exchange of charge-density excitations produced by Friedel oscillations [115]. While most theoretical results on the spin-fluctuation-induced superconductivity are based on RPA-like calculations $[116,117,27,118-124]$, the existence of such an electronic pairing mechanism in a quasi-1D conductor has been recently confirmed by an RG approach [125]. Moreover, it has been recently realized that CDW fluctuations can play an important role in stabilizing a triplet phase [126-128,107,129-131]. Below we discuss in simple terms the link between spin / charge fluctuations and unconventional pairing [124], and present recent results obtained from an RG approach [129-131].

\subsection{Superconductivity from spin and charge fluctuations}

Considering for the time being only intrachain interactions, the interacting part of the Hamiltonian within the $g$-ology framework [108] reads

$$
H_{\mathrm{int}}=\sum_{q}\left[g_{\mathrm{ch}} \rho(-\mathbf{q}) \rho(\mathbf{q})+g_{\mathrm{sp}} \mathbf{S}(-\mathbf{q}) \cdot \mathbf{S}(\mathbf{q})\right]
$$

(from now on we neglect the $c$ axis and consider a 2D-model), where $\rho_{\mathbf{q}}$ and $S_{\mathbf{q}}$ are the charge- and spin-density operators in the Peierls channel $\left(q_{x} \sim 2 k_{F}\right), \quad g_{\mathrm{ch}}=g_{1}-g_{2} / 2 \quad$ and $\quad g_{\mathrm{sp}}=-g_{2} / 2$. Starting from a half-filled extended Hubbard model, we obtain $g_{1}=U-2 V$ and $g_{2}=U+2 V$, where $U$ is the onsite and $V$ the nearest-neighbor lattice site (dimer) interaction. For simplicity, we do not consider Umklapp scattering $\left(g_{3}\right)$, since it does not play an important role in the present qualitative discussion. For repulsive interactions $g_{1} \sim g_{2}>0$, short-range spin fluctuations develop at low temperatures due to the nesting of the Fermi surface. They can be described by an effective Hamiltonian $H_{\text {int }}^{\text {eff }}$ obtained from (7) by replacing the bare coupling constants by their (static) RPA values

$$
\begin{aligned}
& g_{\mathrm{ch}}^{R P A}(\mathbf{q})=\frac{g_{\mathrm{ch}}}{1+g_{\mathrm{ch}} \chi_{0}(\mathbf{q})}=g_{\mathrm{ch}}-g_{\mathrm{ch}}^{2} \chi_{\mathrm{ch}}^{R P A}(\mathbf{q}), \\
& g_{\mathrm{sp}}^{R P A}(\mathbf{q})=\frac{g_{\mathrm{sp}}}{1+g_{\mathrm{sp}} \chi_{0}(\mathbf{q})}=g_{\mathrm{sp}}-g_{\mathrm{sp}}^{2} \chi_{\mathrm{sp}}^{R P A}(\mathbf{q}),(8)
\end{aligned}
$$

where $\chi^{R P A}$ is the static ( $\left.\omega=0\right)$ RPA susceptibility. The bare particle-hole susceptibility diverges at low temperatures, i.e. $\chi_{0}(\mathbf{Q}) \sim \ln \left(E_{0} / \max \left(T, t_{\perp b}^{\prime}\right)\right)$, due to the $\mathbf{Q}=\left(2 k_{F}, \pi\right)$ nesting of the quasi-1D Fermi surface $\left(\varepsilon_{\mathbf{k}}-\mu \simeq-\varepsilon_{\mathbf{k}+\mathbf{Q}}+\mu\right)$. ( $E_{0}$ is a high-energy cutoff of the order of the bandwidth.) The divergence is cut off by deviations from perfect nesting, characterized by the energy scale $t_{\perp b}^{\prime}$ [Eq. (1)]. In the Bechgaard salts $t_{\perp b}^{\prime} \sim 10 \mathrm{~K}$ and varies with pressure.

When the nesting of the Fermi surface is good ( small $t_{\perp b}^{\prime}$ ), the spin susceptibility $\chi_{\mathrm{sp}}^{R P A}(\mathbf{Q})$ diverges at low temperatures, thus signaling the formation of an SDW. A larger value of $t_{\perp b}^{\prime}$ frustrates antiferromagnetism and, when exceeding a threshold value, eliminates the transition to the SDW phase [132,133]. In that case, the (remaining) short-range spin fluctuations can lead to pairing between fermions. To see this, we rewrite the effective Hamiltonian $H_{\text {int }}^{\text {eff }}$ in the particle-particle (Cooper) channel

$$
\begin{aligned}
H_{\text {int }}^{\text {eff }} & =\sum_{\mathbf{k}, \mathbf{k}^{\prime}}\left[g_{s}\left(\mathbf{k}, \mathbf{k}^{\prime}\right) O_{s}^{*}(\mathbf{k}) O_{s}\left(\mathbf{k}^{\prime}\right)+\right. \\
& \left.+g_{t}\left(\mathbf{k}, \mathbf{k}^{\prime}\right) O_{t}^{*}(\mathbf{k}) \cdot O_{t}\left(\mathbf{k}^{\prime}\right)\right]
\end{aligned}
$$

(we consider only Cooper pairs with zero total momentum), where

$$
\begin{gathered}
g_{s}\left(\mathbf{k}, \mathbf{k}^{\prime}\right)=-3 g_{\mathrm{sp}}^{R P A}\left(\mathbf{k}+\mathbf{k}^{\prime}\right)+g_{\mathrm{ch}}^{R P A}\left(\mathbf{k}+\mathbf{k}^{\prime}\right), \\
g_{t}\left(\mathbf{k}, \mathbf{k}^{\prime}\right)=-g_{\mathrm{sp}}^{R P A}\left(\mathbf{k}+\mathbf{k}^{\prime}\right)-g_{\mathrm{ch}}^{R P A}\left(\mathbf{k}+\mathbf{k}^{\prime}\right)
\end{gathered}
$$

are the effective interactions in the singlet and triplet spin pairing channels (Fig. 9). $O_{s}(\mathbf{k})\left(\mathbf{O}_{t}(\mathbf{k})\right)$, is the annihilation operator of a pair $(\mathbf{k},-\mathbf{k})$ in a singlet (triplet) spin state, and $\mathbf{O}_{t}=\left(O_{t}^{1}, O_{t}^{0}, O_{t}^{-1}\right)$ denotes the three components $S^{z}=1,0,-1$ of the triplet state (total spin $S=1$ ).

On the basis of the effective Hamiltonian (9) the BCS theory predicts a superconducting transition whenever the effective interaction $g_{s, t}$ turns out to be attractive in (at least) one pairing channel. A simple argument shows that this is indeed always the case in the presence of short-range spin fluctuations. The spin susceptibility $\chi_{\mathrm{sp}}^{R P A}\left(\mathbf{k}+\mathbf{k}^{\prime}\right)$ exhibits a pronounced peak around $\mathbf{k}+\mathbf{k}^{\prime}=\mathbf{Q}$. Neglecting the unimportant $k_{\|}$ dependence, its Fourier series expansion reads

$$
\begin{gathered}
\chi_{\mathrm{sp}}^{R P A}\left(2 k_{F}, k_{\perp}+k_{\perp}^{\prime}\right)=\sum_{n=0}^{\infty} a_{n}(-1)^{n} \cos \left[n\left(k_{\perp}+k_{\perp}^{\prime}\right)\right]= \\
=\sum_{n=0}^{\infty} a_{n}(-1)^{n}\left[\cos n k_{\perp} \cos n k_{\perp}^{\prime}-\sin n k_{\perp} \sin n k_{\perp}^{\prime}\right],
\end{gathered}
$$




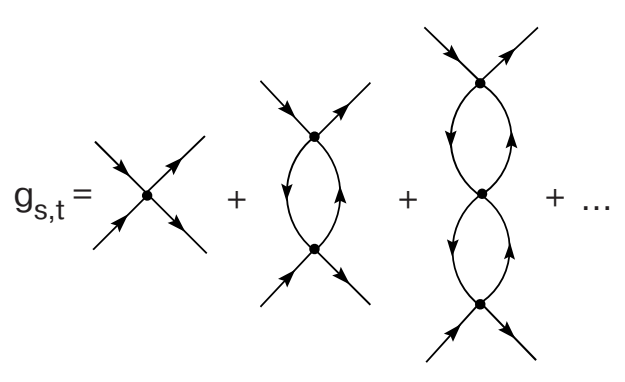

Fig. 9. Diagrammatic representation of the effective interaction $g_{s, t}$ in the Cooper channel within the RPA.

where $a_{n} \geq 0$. Choosing $a_{n}=a_{0}$, one obtains a diverging spin susceptibility $\chi_{\mathrm{sp}}^{R P A}\left(2 k_{F}, k_{\perp}+k_{\perp}^{\prime}\right) \propto$ $\propto \delta\left(k_{\perp}+k_{\perp}^{\prime}-\pi\right)$. The condition $a_{0}>a_{1}>\ldots \geq 0$ gives a broadened peak around $k_{\perp}+k_{\perp}=\pi$. Eqs. $(10,11)$ show that the effective interaction in the singlet channel contains attractive interactions for any value of $n$. In real space, $n$ corresponds to the range of the pairing interaction in the $b$ direction. The dominant attractive interaction corresponds to nearest-neigh-

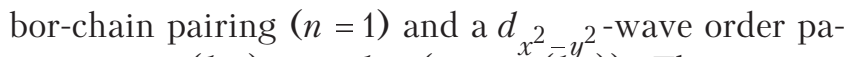
rameter $\Delta_{r}\left(k_{\perp}\right) \sim \cos k_{\perp}\left(r=\operatorname{sgn}\left(k_{x}^{y^{2}}\right)\right)$. The interaction is also attractive in the triplet $f$-wave channel $\left(\Delta_{r}\left(k_{\perp}\right) \sim r \cos k_{\perp}\right)$. However, all the three components of a (spin-one boson) SDW fluctuation contribute to the superconducting coupling in the singlet channel - hence the factor of 3 in the first of equations (10). The latter therefore always dominates over the triplet one when charge fluctuations are not important. Note that the interaction is repulsive in the singlet $d_{x y}$-wave $\left(\Delta_{r}\left(k_{\perp}\right) \sim r \sin k_{\perp}\right)$ and the triplet $p_{y}$-wave $\left(\sin k_{\perp}\right)$ channels.

Equations (10) show that CDW fluctuations tend to suppress the singlet pairing, but reinforce the triplet one. In the Bechgaard salts, the physical relevance of CDW fluctuations has been borne out by the puzzling observation of a CDW that coexists with the SDW (Sec. 2.2.) [33,53,54]. Within the framework of an extended anisotropic Hubbard model, recent RPA calculations have shown that the triplet $f$-wave pairing can overcome the singlet $d_{x^{2}-y^{2}}$-wave pairing when the intrachain interactions are chosen such as to boost the CDW fluctuations with respect to the SDW ones [126-128]. In a half-filled model, this however requires the nearest-neighbor (intrachain) interaction $V$ to exceed $U / 2$. In a quarter-filled model - appropriate if one ignores the weak dimerization along the chains - the condition for $f$-wave superconductivity becomes $V_{2} \geq U / 2-V_{2}$ is the next-nearest-neighbor (intrachain) Coulomb interaction - and appears even more unrealistic. Similar conclusions were reached within an RG approach [107].
Given that electrons interact through the Coulomb interaction, not only intrachain but also interchain interactions are present in practice. At large momentum transfer, the interchain interaction is well known to favor a CDW ordered state [134-137]. This mechanism is mostly responsible for CDW long-range order observed in several organic and inorganic low-dimensional solids (e.g. TTF-TCNQ) [138,139]. In the Bechgaard salts, both the interchain Coulomb interaction and the kinetic interchain coupling $\left(t_{\perp b}\right)$ are likely to be important in the temperature range where superconductivity and SDW instability occur, and should be considered on equal footing. An RG approach has recently been used to determine the phase diagram of an extended quasi-1D electron gas model that includes interchain hopping, nesting deviations and both intrachain and interchain interactions [129-131]. The intrachain interactions turn out to have a sizeable impact on the structure of the phase diagram. Unexpectedly, for reasonably small values of the interchain interactions, the singlet $d_{x^{2}-y^{2}}$-wave superconducting phase is destabilized to the benefit of the triplet $f$-wave phase with a similar range of $T_{c}$. The SDW phase is also found to be close in stability to a CDW phase. Before presenting these results in more detail (Sec. 4.2.), let us discuss in simple terms the role of interchain interactions. The interchain backward scattering amplitude $g_{1}^{\perp}(>0)$ contributes to the effective interaction in the Cooper channel,

$$
\begin{gathered}
g_{s}\left(k_{\perp}, k_{\perp}^{\prime}\right) \rightarrow g_{s}\left(k_{\perp}, k_{\perp}^{\prime}\right)+ \\
+2 g_{1}^{\perp}\left[\cos k_{\perp} \cos k_{\perp}^{\prime}-\sin k_{\perp} \sin k_{\perp}^{\prime}\right], \\
g_{t}\left(k_{\perp}, k_{\perp}^{\prime}\right) \rightarrow g_{t}\left(k_{\perp}, k_{\perp}^{\prime}\right)+ \\
+2 g_{1}^{\perp}\left[-\cos k_{\perp} \cos k_{\perp}^{\prime}+\sin k_{\perp} \sin k_{\perp}^{\prime}\right] .
\end{gathered}
$$

It thus tends to suppress singlet $d_{x^{2}-y^{2}}$ pairing, but favors triplet $f$-wave pairing. In addition to this «direct» contribution, $g_{1}^{\perp}$ reinforces CDW fluctuations,

$$
g_{\mathrm{ch}}\left(q_{\perp}\right) \rightarrow g_{\mathrm{ch}}\left(q_{\perp}\right)+2 g_{1}^{\perp} \cos q_{\perp}
$$

and therefore enhances the $f$-wave pairing over the

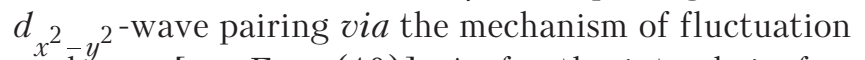
exchange [see Eqs. (10)]. As for the interchain forward scattering $g_{2}^{\perp}$, its direct contribution to the DW channel is negligible, but it has a detrimental effect on both singlet and triplet nearest-neighbor-chain pairings. This latter effect, which is neutralized by the Umklapp scattering processes, can lead to next-nearest-neighbor-chain pairings when Umklapp processes are very weak [131]. 


\section{2. $R G$ calculation of the phase diagram of quasi-1D conductors}

As a systematic and unbiased method with no $a$ priori assumption, the RG method is perfectly suited to study competing instabilities. The zero-temperature phase diagram obtained with this technique is shown in Fig. $10[130,131]$. In the absence of interchain interactions $\left(g_{1}^{\perp}=g_{2}^{\perp}=0\right)$, it confirms the validity of the qualitative arguments given above. When the nesting of the Fermi surface is nearly perfect (small $t_{\perp}^{\prime}$ ) the ground state is an SDW. Above a threshold value of $t_{\perp}^{\prime}$, the low-temperature SDW instability is suppressed and the ground state becomes a $d_{x^{2}-y^{2}}$-wave superconducting ( $\mathrm{SC} d$ ) state with an order parameter $\Delta_{r}\left(k_{\perp}\right) \propto \cos k_{\perp}$ [125]. In the presence of interchain interactions $\left(g_{1}^{\perp}>0\right)$, the region of stability of the $\mathrm{SC} d$ phase shrinks, and a triplet superconducting $f$-wave $(\mathrm{SC} f$ ) phase appears next to the $d$-wave phase for $\tilde{g}_{1}^{\perp}=g_{1}^{\perp} / \pi v_{F} \simeq 0.1-$ obtained here for typical values of intrachain couplings and band parameters [130,131]. For larger values of the interchain interactions, the $\mathrm{SC} d$ phase disappears and the region of stability of the $f$-wave superconducting phase widens. In addition a CDW phase appears, thus giving the sequence of phase transitions $\mathrm{SDW} \rightarrow \mathrm{CDW} \rightarrow \mathrm{SC} f$ as a function of $t_{\perp}^{\prime}$. For $\tilde{g}_{1}^{\perp} \gtrsim 0.12$, the SDW phase disappears. Note that for $\tilde{g}_{1}^{\perp} \simeq 0.11$, the region of stability of the CDW phase is very narrow, and there is essentially a direct transition between the SDW and $\mathrm{SC} f$ phases.

The RG calculations yield $T_{c} \sim 30 \mathrm{~K}$ for the SDW phase in the case of perfect nesting and $T_{c} \sim 0.6-1.2 \mathrm{~K}$ for the superconducting phase, in reasonable agreement with the experimental observations in the Bechgaard salts. Fig. 11 shows the transition temperature $T_{c}$ as a function of $t_{\perp}^{\prime}$ for three different values of

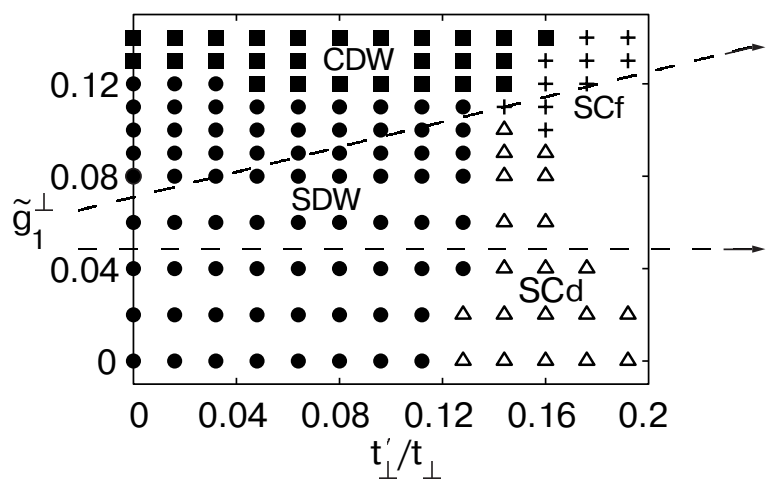

Fig. 10. $T=0$ phase diagram as a function of $t_{\perp}^{\prime} / t_{\perp}$ and $\tilde{g}_{1}^{\perp}$. Circles: SDW, squares: CDW, triangles: SCd $\left(\Delta_{r}\left(k_{\perp}\right) \propto \cos k_{\perp}\right)$, crosses: SCf $\left(\Delta_{r}\left(k_{\perp}\right) \propto r \cos k_{\perp}\right)$. The dashed lines indicate two (among many) possible pressure axes, corresponding to transitions $\mathrm{SDW} \rightarrow \mathrm{SC} d$ and $\mathrm{SDW} \rightarrow \mathrm{SC} f[130,131]$. the interchain interactions, $\tilde{g}_{1}^{\perp}=0,0.11$ and 0.14 , corresponding to the three different sequences of phase transitions as a function of $t_{\perp}^{\prime}: \mathrm{SDW} \rightarrow \mathrm{SC} d$, $\mathrm{SDW} \rightarrow(\mathrm{CDW}) \rightarrow \mathrm{SC} f$ and $\mathrm{CD} \rightarrow \mathrm{WSC} f$. The phase diagram is unchanged when both $g_{2}^{\perp}$ and a weak Umklapp scattering amplitude $g_{3}$ are included [130,131].

The RG approach also provides important information about the fluctuations in the normal phase. The dominant fluctuations above the SC $d$ phase are SDW fluctuations as observed experimentally (Sec. 2.2.). Although they saturate below $T \sim t_{\perp}^{\prime}$ where the $\mathrm{SC} d$ fluctuations become more and more important, the latter dominate only in a very narrow temperature range above the superconducting transition (Fig. 12). Above the SCf and CDW phases, one expects strong CDW fluctuations driven by $g_{1}^{\perp}$. Fig. 13 shows that for $\tilde{g}_{1}^{\perp} \sim 0.11-0.12$, strong SDW and CDW fluctuations coexist above the SC $f$ phase. Remarkably, there are regions of the phase diagram where the SDW fluctuations remain the dominant ones in the normal phase above the SC $f$ or CDW phase (Fig. 13,b).

A central result of the RG calculation is the close proximity of SDW, CDW and SCf phases in the phase diagram of a quasi-1D conductor within a realistic range of values for the repulsive intrachain and interchain interactions. Although this proximity is found only in a small range of interchain interactions, there are several features that suggest that this part of the phase diagram is the relevant one for the Bechgaard salts. i) SDW fluctuations remain important in the normal phase throughout the whole phase diagram. They are the dominant fluctuations above the SCd phase, and remain strong - being sometimes even dominant - above the SC $f$ phase where they coexist with strong CDW fluctuations, in accordance with observations [24,33]. ii) The SCf and CDW phases stand nearby in the theoretical phase diagram, the CDW phase always closely following the SC $f$ phase when the interchain interactions increase. This agrees with the experimental finding that both SDW

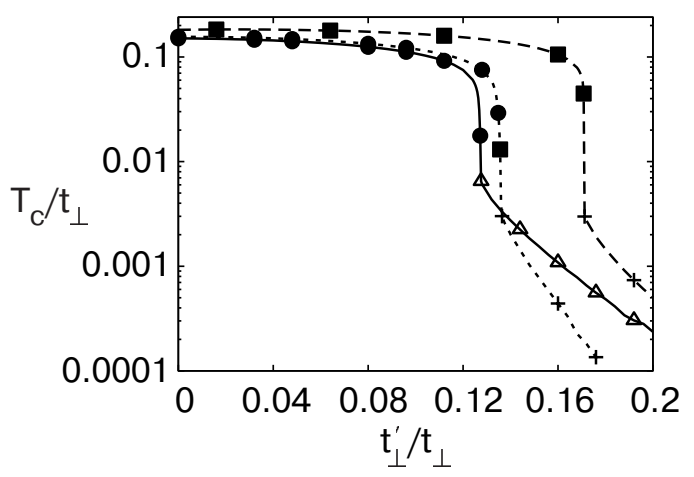

Fig. 11. Transition temperature as a function of $t_{\perp}^{\prime} / t_{\perp}$ for $\tilde{g}_{1}^{\perp}=0,0.11$ and 0.14 , corresponding to solid, dotted, and dashed lines, respectively [130,131]. 


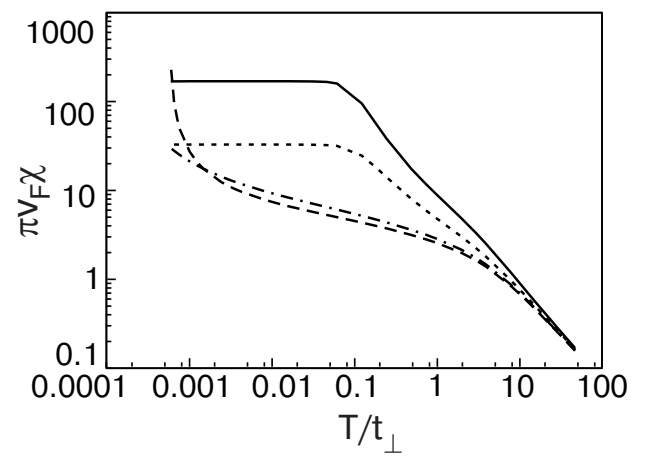

Fig. 12. Temperature dependence of the susceptibilities in the normal phase above the SC $d$ phase $\left(t_{\perp}^{\prime}=0.152 t_{\perp}\right.$ and $\left.\tilde{g}_{1}^{\perp}=0.08\right)$. The continuous, dotted, dashed, and dashed-dotted lines correspond to $\mathrm{SDW}, \mathrm{CDW}, \mathrm{SC} d$ and SC $f$ correlations, respectively [130,131].

and CDW coexist in the DW phase of the Bechgaard salts $[53,54]$ and the existence, besides SDW correlations, of CDW fluctuations in the normal state above the superconducting phase [33]. iii) Depending how one moves in practice in the phase diagram as a function of pressure, these results are compatible with either a singlet $d_{x^{2}-y^{2}}$-wave or a triplet $f$-wave superconducting phase in the Bechgaard salts (see the two pressure axes in Fig. 10). Moreover, one cannot exclude that both SCd and SC $f$ phases exist in these materials, with the sequence of phase transitions $\mathrm{SDW} \rightarrow \mathrm{SC} d \rightarrow \mathrm{SC} f$ as a function of pressure. It is also
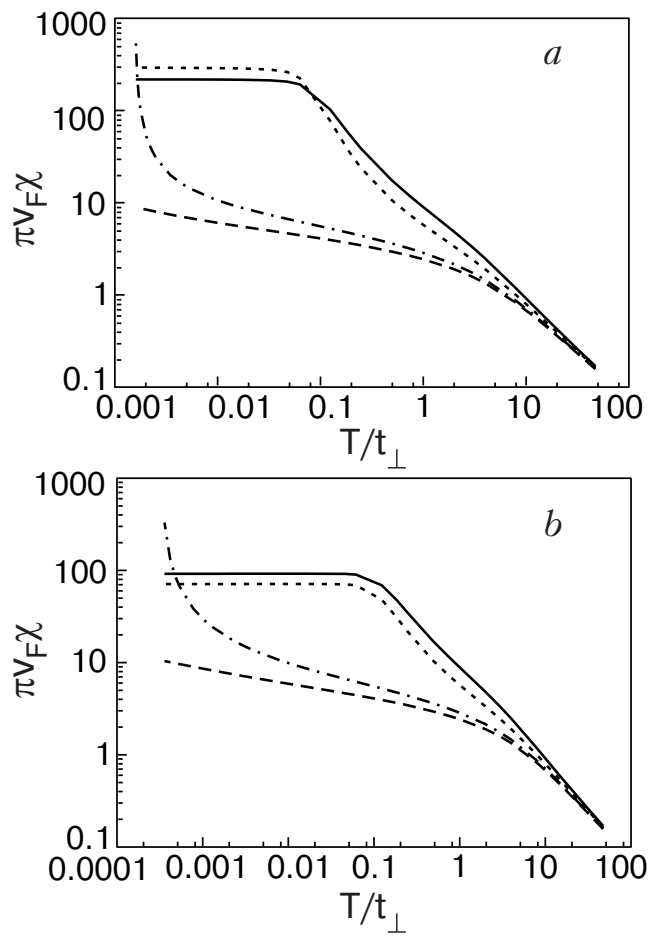

Fig. 13. Temperature dependence of the susceptibilities in the normal phase above the SCf phase for $\tilde{g}_{1}^{\perp}=0.12$, $t_{\perp}^{\prime}=0.152 t_{\perp}(a)$ and $t_{\perp}^{\prime}=0.176 t_{\perp}(b)$. possible that the $\mathrm{SC} f$ phase is stabilized by a magnetic field [140], since an equal-spin pairing triplet phase is not sensitive to the Pauli pair breaking effect contrary to the $\mathrm{SC} d$ phase. This would make possible the existence of large upper critical fields exceeding the Pauli limit [76,78], and would also provide an explanation for the temperature independence of the NMR Knight shift in the superconducting phase [94].

\section{Conclusion}

Notwithstanding the recent experimental progresses, many of the basic questions related to superconductivity in the Bechgaard and Fabre salts remain largely open. The very nature of the superconducting state - the orbital symmetry of the order parameter and the singlet/triplet character of the pairing - is still not known without ambiguity even though recent upper critical field measurements [76-78] and NMR experiments [94,95] support a triplet pairing.

We argued that the conventional electron-phonon mechanism is unable to explain the origin of the superconducting phase. On the other hand, the proximity of the SDW phase, as well as the observation of strong spin fluctuations in the normal state precursor to the superconducting phase $[16,17,24]$, strongly suggest an intimate relationship between antiferromagnetism and superconductivity in the Bechgaard/Fabre salts. The scenario originally proposed by Emery [114], whereby short-range $\mathrm{AF}$ spin fluctuations can give rise to anisotropic pairing and thus stabilize a superconducting phase, is so far the only one that is consistent with the experimental observations and the repulsive nature of the electron-electron interactions.

Within the framework of the RG approach, it has recently been shown that when spin and charge fluctuations are taken into account on equal footing, both singlet $d_{x^{2}-y^{2}}$ - and triplet $f$-wave superconducting phases can emerge at low temperatures whenever the nesting properties of the Fermi surface deteriorate under pressure [126-128,107,129-131]. CDW fluctuations are enhanced by the long-range part of the $\mathrm{Cou}^{-}$ lomb interaction. Remarkably, for a reasonably small value of the interchain interactions, the singlet

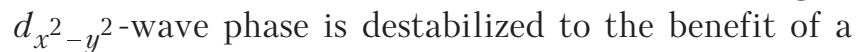
triplet $f$-wave with a similar range of $T_{c}$ [130,131]. The physical relevance of CDW fluctuations in the Bechgaard salts has been born out by the observation of a CDW that actually coexists with the SDW [53,54]. CDW fluctuations were also observed in the normal state precursor to the superconducting state [33].

As a systematic and unbiased method with no $a$ priori assumptions, the RG has proven to be a method of choice to study the physical properties of quasi-1D organic conductors. An important theoretical issue is 
now to go beyond the instabilities of the normal state. On the one hand, the RG analysis should be extended to the low-temperature broken-symmetry phases in order to study the possible coexistence of superconductivity and antiferromagnetism, as well as CDW and SDW, as observed in the Bechgaard salts $[86,88,53,54]$. On the other hand, the RG technique might also enable to tackle the unusual properties of the metallic phase. A recent RG analysis [107] of the AF spin susceptibility in the normal phase has shown that below the dimensional crossover temperature, it differs significantly from the prediction of single-channel (RPA) theories. The interplay between the superconducting and Peierls channels, which is at the origin of spin-fluctuation induced superconductivity, might also be responsible for the unusual properties of the metallic state below the dimensional crossover temperature.

1. D. Jérome, A. Mazaud, M. Ribault, and K. Bechgaard, J. Phys. Lett. (Paris) 41, L95 (1980).

2. D. Jérome and H.J. Schulz, Adv. Phys. 31, 299 (1982).

3. T. Ishiguro and K. Yamaji, Organic Superconductors, of Springer-Verlag Series in Solid-State Science, Springer-Verlag, Berlin, Heidelberg (1990), v. 88.

4. R. H. McKenzie, Comments Cond. Matt. Phys. 18, 309 (1998).

5. S. Lefebvre et al., Phys. Rev. Lett. 85, 5420 (2000).

6. J. Flouquet, arXiv: cond-mat/0501602 (unpublished).

7. D. Jérome, Chem. Rev. 104, 5565 (2004).

8. K. Bechgaard et al., Solid State Commun. 33, 1119 (1980).

9. C. Bourbonnais and D. Jérome, in: Advances Synthetic Metals, Twenty Years of Progress in Science and Technology, P. Bernier, S. Lefrant, and G. Bidan (eds.), Elsevier, New York (1999), p. 206, arXiv: cond-mat/9903101.

10. P M. Grant, J. Phys. (Paris.) 44, 847 (1983).

11. K. Yamaji, J. Phys. Soc. Jpn. 51, 2787 (1982).

12. L. Ducasse et al., J. Phys. C19, 3805 (1986).

13. L. Balicas et al., J. Phys. (France) 4, 1539 (1994).

14. V.J. Emery, R. Bruisma, and S. Baršiić, Phys. Rev. Lett. 48, 1039 (1982).

15. S. Barišić and S. Brazovskii, in: Recent Developments in Condensed Matter Physics, J.T. Devreese (ed.), Plenum, New York, (1981), v. 1, p. 327.

16. D. Jaccard et al., J. Phys.: Cond. Matt. 13, L89 (2001).

17. H. Wilhelm et al., Eur. Phys. J. B21, 175 (2001).

18. T. Adachi et al., J. Am. Chem. Soc. 122, 3238 (2000).

19. C. Bourbonnais and D. Jérome, Science 281, 1156 (1998).

20. F.D.M. Haldane, J. Phys. C14, 2585 (1981).

21. T. Giamarchi, Quantum Physics in One Dimension, Oxford University Press, Oxford (2004).

22. C. Coulon et al., J. Phys. (Paris) 43, 1059 (1982).

23. A. Schwartz et al., Phys. Rev. B58, 1261 (1998).
24. P. Wzietek et al., J. Phys. 3, 171 (1993).

25. C. Bourbonnais et al., Phys. Rev. Lett. 62, 1532 (1989).

26. C. Bourbonnais, J. Phys. 3, 143 (1993).

27. C. Bourbonnais and L.G. Caron, Europhys. Lett. 5, 209 (1988).

28. C. Bourbonnais and L.G. Caron, Int. J. Mod. Phys. B5, 1033 (1991).

29. S.E. Brown et al., Synth. Metals 86, 1937 (1997).

30. C. Bourbonnais, F. Creuzet, D. Jérome, and K. Bechgaard, J. Phys. Lett. (Paris) 45, L755 (1984).

31. C.S. Jacobsen, D.B. Tanner, and K. Bechgaard, Phys. Rev. Lett. 46, 1142 (1981).

32. C.S. Jacobsen, D.B. Tanner, and K. Bechgaard, Phys. Rev. B28, 7019 (1983).

33. N. Cao, T. Timusk, and K. Bechgaard, J. Phys. 6, 1719 (1996).

34. M. Dressel, A. Schwartz, G. Grüner, and L. Degiorgi, Phys. Rev. Lett. 77, 398 (1996).

35. T. Giamarchi, Physica B230-232, 975 (1997).

36. V. Vescoli et al., Science 281, 1181 (1998).

37. T. Giamarchi, Chem. Rev. 104, 5037 (2004).

38. J. Favand and F. Mila, Phys. Rev. B54, 10425 (1996).

39. J. Moser et al., Phys. Rev. Lett. 84, 2674 (2000).

40. P. Auban-Senzier, D. Jérome, and J. Moser, in: Physical Phenomena at High Magnetic Fields, Z. Fisk, L. Gor'kov, and L. Schrieffer (eds.), World Scientic, Singapore (1999).

41. G. Mihály, I. Kézsmárki, F. Zámborszky, and L. Forro, Phys. Rev. Lett. 84, 2670 (2000).

42. P. Fertey, M. Poirier, and P. Batail, Eur. Phys. J. B10, 305 (1999).

43. J. Moser et al., Eur. Phys. J. B1, 39 (1998).

44. F. Zwick et al., Phys. Rev. Lett. 79, 3982 (1997).

45. B. Dardel et al., Europhys. Lett. 24, 687 (1993).

46. A. Georges, T. Giamarchi, and N. Sandler, Phys. Rev. B61, 16393 (2000).

47. G.M. Danner, W. Kang, and P.M. Chaikin, Phys. Rev. Lett. 72, 3714 (1994).

48. L.P. Gor'kov, J. Phys. (France) 6, 1697 (1996).

49. A.T. Zheleznyak and V.M. Yakovenko, Eur. Phys. J. B11, 385 (1999).

50. P.M. Chaikin, J. Phys. (France) 6, 1875 (1996).

51. P. Lederer, J. Phys. (France) 6, 1899 (1996).

52. V.M. Yakovenko and H.S. Goan, J. Phys. 6, 1917 (1996).

53. J.P. Pouget and S. Ravy, J. Phys. 6, 1501 (1996).

54. S. Kagoshima et al., Solid State Commun. 110, 479 (1999).

55. H. Seo and H. Fukuyama, J. Phys. Soc. Jpn. 66, 1249 (1997).

56. N. Kobayashi, M. Ogata, and K. Yonemitsu, J. Phys. Soc. Jpn. 67, 1098 (1998).

57. S. Mazumdar, S. Ramasesha, R. T. Clay, and D.K. Campbell, Phys. Rev. Lett. 82, 1522 (1999).

58. Y. Tomio and Y. Suzumura, J. Phys. Soc. Jpn. 69, 796 (2000). 
59. Y. Tomio and Y. Suzumura, J. Phys. Chem. Solids 62, 431 (2001).

60. P. Garoche, R. Brusetti, and K. Bechgaard, Phys. Rev. Lett. 49, 1346 (1982).

61. P. Garoche, R. Brusetti, and D. Jérome, J. Phys. Lett. (Paris) 43, L147 (1982).

62. D.L. Gubser et al., Phys. Rev. B24, 478 (1981).

63. K. Murata et al., Mol. Cryst. Liq. Cryst. 79, 639 (1982)

64. R.L. Green, P. Haen, S.Z. Huang, and E.M. Engler, Mol. Cryst. Liq. Cryst. 79, 183 (1982)

65. R. Brusetti, M. Ribault, D. Jérome, and K. Bechgaard, J. Phys. (France) 43, 52 (1982).

66. M.Y. Choi et al., Phys. Rev. B25, 6208 (1985)

67. S. Bouffard et al., J. Phys. C15, 2951 (1982).

68. A.A. Abrikosov, JETP Lett. 37, 503 (1983).

69. C. Coulon et al., J. Phys. (France) 43, 1721 (1982).

70. S. Tomic et al., J. Phys. Coll. (Paris) 44, C3 (1983).

71. N. Joo et al., Eur. Phys. J. B40, 43 (2004).

72. Q. Yuan et al., Phys. Rev. B68, 174510 (2003).

73. L.P. Gor'kov and D. Jérome, J. Phys. Lett. (Paris) 46, L643 (1985).

74. A.M. Clogston, Phys. Rev. Lett. 9, 266 (1962).

75. B.S. Chandrasekhar, Appl. Phys. Lett. 1, 7 (1962).

76. I.J. Lee, M.J. Naughton, G.M. Danner, and P.M. Chaikin, Phys. Rev. Lett. 78, 3555 (1997).

77. I.J. Lee, P.M. Chaikin, and M.J. Naughton, Phys. Rev. B62, R14669 (2000).

78. J.I. Oh and M.J. Naughton, Phys. Rev. Lett. 92, 067001 (2004).

79. A.G. Lebed, JETP Lett. 44, 114 (1986).

80. L.I. Burlachkov, L.P. Gor'kov, and A.G. Lebed, Europhys. Lett. 4, 941 (1987).

81. N. Dupuis, G. Montambaux, and C.A.R. Sá de Melo, Phys. Rev. Lett. 70, 2613 (1993).

82. N. Dupuis and G. Montambaux, Phys. Rev. B49, 8993 (1994).

83. N. Dupuis, Phys. Rev. B51, 9074 (1995).

84. R.L. Greene and E.M. Engler, Phys. Rev. Lett. 45, 1587 (1980).

85. R. Brusetti, M. Ribault, D. Jérome, and K. Bechgaard, J. Phys. (France) 43, 801 (1982).

86. T. Vuletić et al., Eur. Phys. J. B25, 319 (2002).

87. I.J. Lee, P.M. Chaikin, and M.J. Naughton, Phys. Rev. Lett. 88, 207002 (2002).

88. I.J. Lee et al., Phys. Rev. Lett. 94, 197001 (2005).

89. A.I. Larkin and Y.N. Ovchinnikov, Sov. Phys. JETP 20, 762 (1965).

90. P. Fulde and R.A. Ferrell, Phys. Rev. 135, A550 (1965)

91. A.I. Buzdin and S.V. Polonskii, Sov. Phys. JETP 66, 422 (1983)

92. A.G. Lebed, Phys. Rev. B59, R721 (1999).

93. A.G. Lebed, K. Machida, and M. Ozaki, Phys. Rev. B62, R795 (2000).

94. I.J. Lee et al., Phys. Rev. Lett. 88, 017004 (2002).

95. I. J. Lee et al., Phys. Rev. B68, 092510 (2003).

96. M. Takigawa, H. Yasuoka, and G. Saito, J. Phys. Soc. Jpn. 56, 873 (1987).
97. D. Jérome and C. R. Pasquier, in: Superconductors, A.V. Narlikar (ed.), Springer-Verlag, Berlin (2005).

98. P. Fulde and K. Maki, Phys. Rev. B139, A788 (1965).

99. K. Sengupta et al., Phys. Rev. B63, 144531 (2001).

100. Y. Tanuma, K. Kuroki, Y. Tanaka, and S. Kashiwaya, Phys. Rev. B64, 214510 (2001).

101. Y. Tanuma et al., Phys. Rev. B66, 094507 (2002).

102. Y. Tanuma, K. Kuroki, Y. Tanaka, and S. Kashiwaya, Phys. Rev. B68, 214513 (2003).

103. H.I. Ha, J.I. Oh, J. Moser, and M.J. Naughton, Synth. Metals 137, 1215 (2003).

104. M. Naughton, private communication (unpublished).

105. S. Belin and K. Behnia, Phys. Rev. Lett. 79, 2125 (1997).

106. H. Shimahara, Phys. Rev. B61, R14936 (2000).

107. Y. Fuseya and Y. Suzumura, J. Phys. Soc. Jpn. 74, 1264 (2005).

108. J. Sólyom, Adv. Phys. 28, 201 (1979).

109. V.J. Emery, J. Phys. Coll. (Paris) 44, C3 (1983).

110. S. Barišić, J. Labbé, and J. Friedel, Phys. Rev. Lett. 25, 99 (1970).

111. W.P. Su, J.R. Schrieffer, and A.J. Heeger, Phys. Rev. Lett. 42, 1698 (1979).

112. F. Creuzet et al., Synth. Metals 19, 289 (1987).

113. C. Bourbonnais and B. Dumoulin, J. Phys. 6, 1727 (1996)

114. V.J. Emery, Synth. Metals 13, 21 (1986).

115. W. Kohn and J.M. Luttinger, Phys. Rev. Lett. 15, 524 (1965).

116. M.T. Béal-Monod, C. Bourbonnais, and V.J. Emery, Phys. Rev. B34, 7716 (1986).

117. L.G. Caron and C. Bourbonnais, Physica B143, 453 (1986).

118. D.J. Scalapino, E. Loh, and J.E. Hirsch, Phys. Rev. B34, 8190 (1986).

119. D.J. Scalapino, E. Loh, and J.E. Hirsch, Phys. Rev. B35, 6694 (1987).

120. K. Miyake, S. Schmitt-Rink, and C.M. Varma, Phys. Rev. B34, 6554 (1986).

121. H. Shimahara, J. Phys. Soc. Jpn. 58, 1735 (1988).

122. H. Kino and H. Kontani, J. Low Temp. Phys. 117, 317 (1999).

123. K. Kuroki and H. Aoki, Phys. Rev. B60, 3060 (1999).

124. D.J. Scalapino, Phys. Rep. 250, 329 (1995).

125. R. Duprat and C. Bourbonnais, Eur. Phys. J. B21, 219 (2001).

126. K. Kuroki, R. Arita, and H. Aoki, Phys. Rev. B63, 094509 (2001).

127. S. Onari, R. Arita, K. Kuroki, and H. Aoki, Phys. Rev. B70, 94523 (2004).

128. Y. Tanaka and K. Kuroki, Phys. Rev. B70, R060502 (2004).

129. C. Bourbonnais and R. Duprat, Bull. Am. Phys. Soc. 49, 1:179 (2004).

130. J.C. Nickel, R. Duprat, C. Bourbonnais, and N. Dupuis, Phys. Rev. Lett. 95, 247001 (2005).

131. J.C. Nickel, R. Duprat, C. Bourbonnais, and N. Dupuis, arXiv: cond-mat/0510744 (unpublished). 
132. Y. Hasegawa and H. Fukuyama, J. Phys. Soc. Jpn. 55, 3978 (1986).

133. G. Montambaux, Phys. Rev. B38, 4788 (1988).

134. L.P. Gor'kov and I.E. Dzyaloshinskii, Sov. Phys. JETP 40, 198 (1974).

135. L. Mihály and J. Sólyom, J. Low Temp. Phys. 24, 579 (1976).

136. P.A. Lee, T.M. Rice, and R.A. Klemm, Phys. Rev. B15, 2984 (1977).
137. N. Menyhárd, Solidi State Commun. 21, 495 (1977).

138. S. Barišić and A. Bjeliš, in: Theoretical Aspects of Band Structures and Electronic Properties of Pseudo-One-Dimensional Solids, H. Kaminura (ed.), D. Reidel, Dordrecht (1985), p. 49.

139. J.P. Pouget and R. Comes, in: Charge Density Waves in Solids, L.P. Gor'kov and G. Grüner (eds.), Elsevier Science, Amsterdam (1989), p. 85.

140. H. Shimahara, J. Phys. Soc. Jpn. 69, 1966 (2000). 